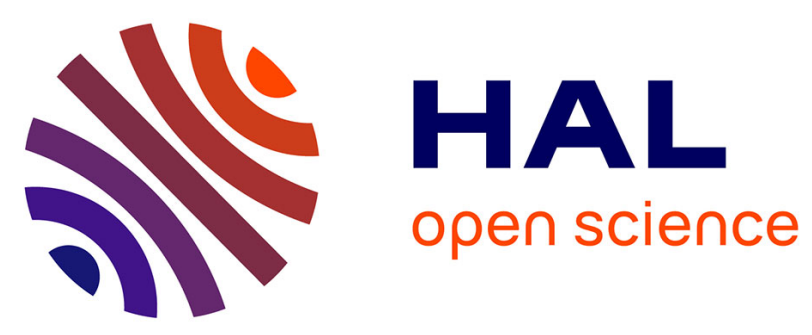

\title{
Optimal Charging Strategy to Minimize Electricity Cost and Prolong Battery Life of Electric Bus Fleet
}

\author{
Adnane Houbbadi, Eduardo Redondo-Iglesias, Rochdi Trigui, Serge Pelissier, \\ Tanguy Bouton
}

\section{- To cite this version:}

Adnane Houbbadi, Eduardo Redondo-Iglesias, Rochdi Trigui, Serge Pelissier, Tanguy Bouton. Optimal Charging Strategy to Minimize Electricity Cost and Prolong Battery Life of Electric Bus Fleet. 2019 IEEE Vehicle Power and Propulsion Conference (VPPC), Oct 2019, Hanoi, France. p. 1-6, 10.1109/VPPC46532.2019.8952493 . hal-02476452

\section{HAL Id: hal-02476452 \\ https://hal.science/hal-02476452}

Submitted on 12 Feb 2020

HAL is a multi-disciplinary open access archive for the deposit and dissemination of scientific research documents, whether they are published or not. The documents may come from teaching and research institutions in France or abroad, or from public or private research centers.
L'archive ouverte pluridisciplinaire HAL, est destinée au dépôt et à la diffusion de documents scientifiques de niveau recherche, publiés ou non, émanant des établissements d'enseignement et de recherche français ou étrangers, des laboratoires publics ou privés. 


\title{
Optimal charging strategy to minimize electricity cost and prolong battery life of electric bus fleet
}

\author{
Adnane Houbbadi* ${ }^{* 1}$, Eduardo Redondo-Iglesias ${ }^{1}$, Rochdi Trigui ${ }^{1}$, Serge Pelissier ${ }^{1}$, \\ Tanguy Bouton ${ }^{2}$ \\ ${ }^{1}$ Univ Lyon, IFSTTAR, AME, ECO7, F-69500 Bron, France \\ ${ }^{2}$ TRANSDEV GROUP, F-92130 Issy-les-Moulineaux, France \\ *Corresponding author: adnane.houbbadi@ifsttar.fr
}

\begin{abstract}
Smart charging is becoming an important and indispensable asset for electric bus fleet to become economically competitive. This paper proposes an innovative approach for setting overnight charging schedules of electric bus fleet. This approach uses nonlinear programming in order to minimize both the electricity cost and the battery aging. The optimization is constrained by the operating buses conditions, the electric vehicle supply equipment, and the power grid. A comparison between the nonlinear programming results and non-dominated sorted genetic algorithm (NSGA-II) will show the difference and complementarities of both approaches and proposes a number of trade-off optimal solutions.
\end{abstract}

Keywords-multi-objective optimization problem (MOP), battery aging, electric vehicles (EVs), electric buses (EBs), Pareto front, non-dominated sorted genetic algorithm (NSGA), nonlinear programming (NLP), two phase method (TPM), fleet operator (FO)

\section{INTRODUCTION}

Electro-mobility in urban public transport is set to play a larger role to reduce greenhouse emissions and local pollutants. However, as a result of a possible large-scale deployment of electric buses (EBs), the power grid should be strengthened to avoid the overloading, the peak-demand and network congestion problems [1]. On the other hand, the EBs purchase costs should be mitigated by low operational costs.

Many fleet operators (FO) are increasingly interested in the viability of the electric bus system. Therefore, new methods are required to optimize the operation cost of $\mathrm{EB}$ depots substation. To this end, the EVs smart charging is one of the main tools to be deployed. Regarding centralized overnight charging for large and small-scale EVs fleets, several optimization methods (e.g. dynamic programming, non-linear programming, (meta)heuristic optimization) were compared through an extensive literature review [2]. Some studies [3-11] have investigated EBs fleets with overnight charging on centralized bus depots. Nevertheless, they analyzed large and small-scale bus depots mostly from the perspective of minimizing the operational costs or the load peak without taking into account the aging of batteries, while using a mono-objective optimization algorithms. In this work, we investigate more precisely the battery electro-thermal and aging behavior of a large-scale EBs fleet while handling a multi-objective optimization problem. In our previous work [12], NSGA-II method has been tested with success for sloving multiobjective problem. The main drawback was the processing time and the possibility of considering only a weak number of buses. In this work, a nonlinear programming (NLP) algorithm has been used to cope with non-linear problems such as battery aging while ensuring a reasonable processing time that will allow to deal with substantial number of buses.

The remainder of the paper proceeds as follows. In Section II, we introduce our proposed approach for smart charging strategy of EBs and the system modeling. In Section III, we detail our approach used for multi-objective optimization problem and we present the mathematical formulation of the problem. Section IV presents the case study of an EB that operates during the day an existing conventional bus line. The optimization takes place during the overnight charging. We present and discuss our results in Section V. The results of the optimization problem will be compared to the previous NSGAII algorithm taken as a reference for a small number of buses [12]. Finally, Section VI draws some conclusions and introduces future work.

\section{PROPOSED APPROACH \& SYSTEM MODELING}

\section{A. Multi-objective optimization methodology for EBs fleet charging}

This part introduces a methodological approach (Fig. 1) for the management of EBs fleet overnight charging. The optimization methodology has been developed in Matlab/Simulink environment. This approach uses NLP to find an optimal charging power profile for each bus depending on objectives and respecting all the constraints while minimizing the charging cost and battery aging cost as well. The fitness function is evaluated using a converter and a battery-coupled model. The optimization takes into account several constraints:

- Operating constraints (number of buses, initial state of charge (SoC) and battery temperature $(\mathrm{T})$, the targeted SoC, arrival and departure time, maintenance period)

- Charging station constraints (number of charging points, maximum charging power...)

- Power grid constraints (time-of-use, power peak demand) 


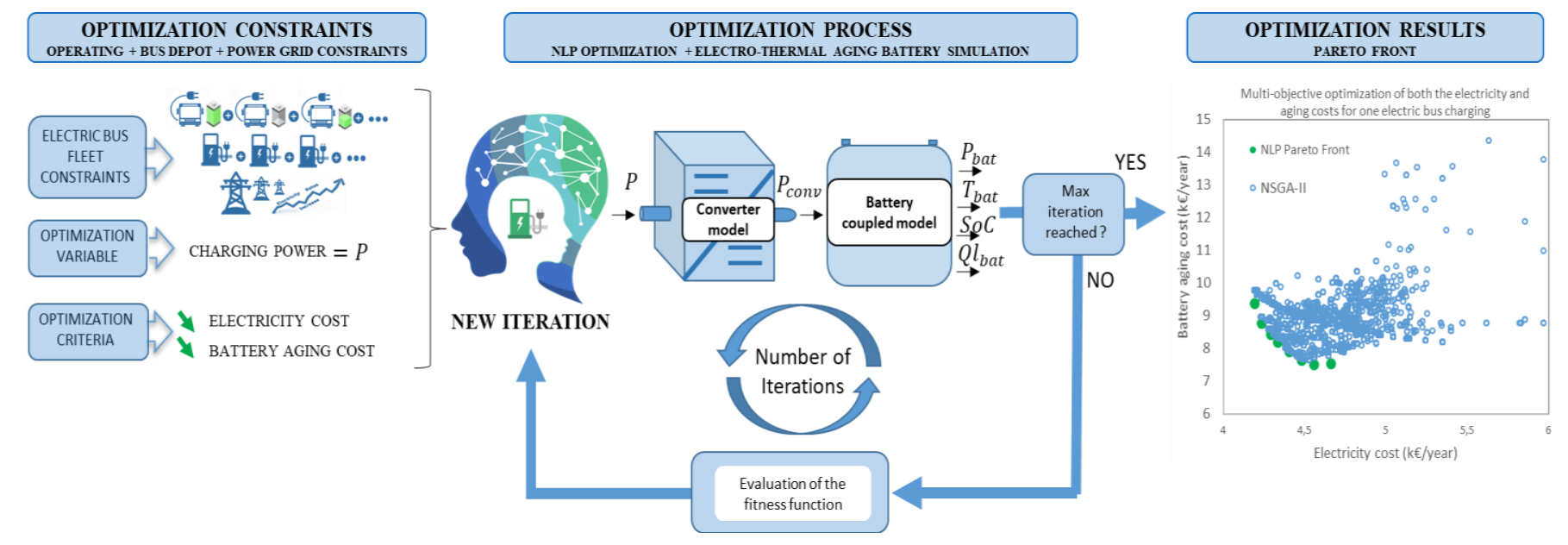

Fig. 1. Multi-objective optimization methodology for the management of EBs fleet charge [13]

\section{B. Coupled Electro-Thermal Aging based battery model}

To make the entire battery model complete and more accurate, three sub-models are coupled in Fig. 2. We used an electric model based on an equivalent electrical circuit previously developed on VEHLIB [14]. The battery thermal model used is a simple model of a prismatic lithium-ion battery (LiFePO4/graphite) based on an equivalent electrical circuit [15]. The equivalent electric circuit equation is given by:

$$
U_{b a t}=O C V-R_{e q} \times I_{b a t}
$$

This model implements an ideal voltage source $O C V$ that represents the open circuit voltage of the battery, and a resistor $R_{e q}$ that includes an ohmic, double layer and diffusion resistance. $I_{b a t}$ is the battery current with a positive value when discharging and a negative value when charging. $U_{b a t}$ is the battery voltage.

The temperature change of the battery is the effect of heat generated by the battery and the heat dissipated from the battery.

$$
C_{t h} \frac{d T_{b a t}}{d t}=I_{b a t}\left(U_{b a t}-O C V\right)-h\left(T_{b a t}-T_{a m b}\right)
$$

Where $I_{b a t}$ is the battery charge current, $U_{b a t}$ is the battery voltage, $O C V$ is the open circuit voltage, $C_{t h}$ is the specific thermal capacity of the battery.

The heat capacity and heat transfer coefficient were estimated by experiments [16] and were compared with experimental values from the literature. As the battery thermal characteristics are strongly dependent on the battery pack configuration, we assumed that we have a configuration similar to that of [16], homogeneous temperature in the battery pack, with no thermal insulation, no forced air convection and no cooling system. The battery pack electrothermal and aging characteristics are presented in Table II. Battery aging mechanisms depend on battery conditions $(T$,
SoC) and battery current (I). Over the time, battery's performances (power, capacity) decay and can be categorized into calendar $(I=0)$ and cycling aging $(I \neq 0)$. In this work, it must be highlighted that cycling aging could be neglected over the period of slow charging as current is very low $(\mathrm{C}$ - rate $\leq \mathrm{C} / 6)$ and the ambient temperature is set to $25^{\circ} \mathrm{C}$ [17]. As for battery calendar aging evaluation, we used an empirical model proposed by [18] to describe the calendar capacity loss as a function of time.

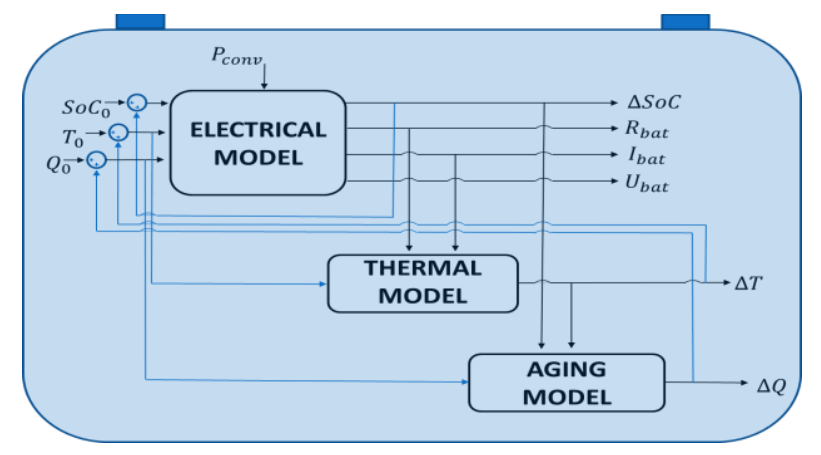

Fig. 2. Coupled Electro-Thermal Aging based battery model

The formula of this semi-empirical model is based on the Eyring equation:

$$
\dot{Q}_{\text {loss }}=A \cdot \exp \left(-\frac{E_{a}}{k T}+B \cdot Q_{a}\right)
$$

Here, $\dot{Q}_{\text {loss }}$ is the capacity loss rate (p.u./day), $A$ is the preexponential factor (p.u./day), $E_{a}$ represents the activation energy for the reaction $(\mathrm{eV}), k$ the Boltzmann constant $(\mathrm{eV} / \mathrm{K}), T$ the absolute temperature $(\mathrm{K}), B$ the quantity of charge factor (no units), $Q_{a}$ the available quantity of charge (p.u.) and $t$ time (day). All the aging parameters values are presented in Table II. The capacity loss is determined by integrating (1) with a 30 min time step. 


\section{MULTI-OBJECTIVE OPTIMIZATION PROBLEM FORMULATION}

Multi-objective optimization is an area of multiple criteria decision making that is concerned with mathematical optimization problems involving more than one objective function to be optimized simultaneously. A multi-objective optimization problem (MOP) can be defined by:

Optimize $\int f(x)=\left(f_{1}(x), f_{2}(x), \ldots, f_{n}(x)\right) x \in F$

Where $n$ is the number of objectives $(n \geq 2), x=\left(x_{1}, x_{2}, . . x_{k}\right)$ is the vector representing the decision variables, $F$ represents the set of feasible solutions and each of the functions $f_{i}(x)$ represents a particular objective. Unlike single-objective optimization problems, the solution to this problem is a set of non-dominated solutions, known as the Pareto front [19].

\section{A. Approach to solve multi-objective problems}

Several approaches for solving multi-objective NP-hard ${ }^{\mathrm{a}}$ problems optimization exist. We can classify the optimization methods into two major approaches:

- Transformation of MOP into mono-objective problems (weighted aggregation method, two-phase method, $\varepsilon$ constraint method...)

\section{- Multi-objectives Pareto approach}

In this work, we will focus on the transformation of MOP into a mono-objective problem. In a first step, we used the first phase of the two-phase method (TPM) that solves a series of mono-objective problems with NLP. The monoobjective problems are obtained by performing a linear weighting of the two objectives. The method begins by finding the extreme points (Fig. 3.a) by solving optimally each objective, and then sub problems are created by weighting the objective functions $\alpha_{1} f_{1}$ and $\alpha_{2} f_{2}$ (Fig. 3.b). The method is then iterated (Fig. 3.c) via a dichotomic search. We then obtain optimal Pareto set points (Fig. 3.d) [20,21].

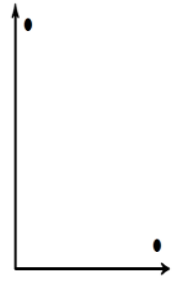

(a)

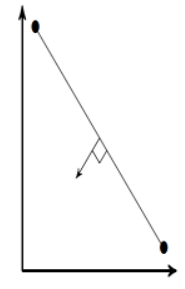

(b)

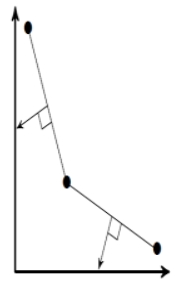

(c)

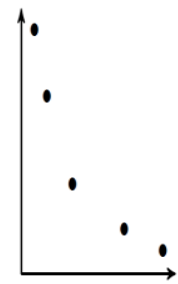

(d)
Fig. 3. Multi-objective proposed optimization method [20]

In this work, we used the two-phase method (TPM) to solve the multi-objective problem. The results obtained will be compared to NSGA-II method which performs a real multiobjective optimization.

\section{B. Representation and evaluation}

In this work, the optimization variable represents the charging power $P$ of the bus fleet in an $(n \times m)$ matrix:

aNP-hard: NP-hard problems are problems for which there is no known polynomial algorithm, so that time to find a solution grows with problem size. $\underline{\text { Optimization variable }}$

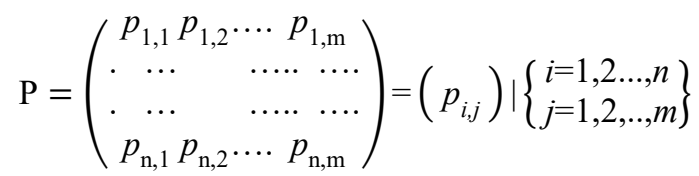

Where $n$ is the total number of EB and $m$ the number of time slots, $p_{i, j}$ is the charging power for EB number $i$ during a time $\operatorname{slot} j$.

\section{Objective function}

The aim is to minimize the overall cost of charging as well as the battery aging cost. This problem can be formulated as follows :

$$
\min \sum_{\mathrm{i}=1}^{\mathrm{n}} \sum_{\mathrm{j}=1}^{\mathrm{m}} \boldsymbol{\alpha}_{1}\left(p_{i, j} \cdot \Delta t . E c_{j}\right)+\boldsymbol{\alpha}_{2}\left(\frac{\Delta Q_{\text {loss }_{i, j}}}{Q_{E o L}} . \text { Bat }_{\text {price }} \cdot E_{\text {bat } i, j}\right)
$$

The charging cost is expressed as a linear function, where $p_{i, j}$ denotes the charging power of EB number $i$ during a time slot $j(\mathrm{~kW}), \Delta t$ represents the time-slot of $30 \mathrm{~min}(\mathrm{~h}), E c_{j}$ the electricity cost during a time slot $j(€ / \mathrm{kWh})$. The battery aging cost represents the cost of replacing the battery spread over the battery lifetime. This cost is expressed as an nonlinear function where $\Delta Q_{\text {loss }_{i, j}}$ is the capacity loss rate of EB number $i$ during a time slot $j$ (p.u./day), $Q_{E o L}$ is the capacity loss at end of life (p.u.), Bat price $_{\text {is }}$ the price of the LiFePO4 battery $(€ / \mathrm{kWh})$ and $E_{\text {bat } i, j}$ is the amount of energy of EB number $i$ required during a time $\operatorname{slot} j(\mathrm{kWh}), \alpha_{1}$ and $\alpha_{2}$ represent the weighting factors that define the search direction.

\section{Linear equality and inequality constraints}

During optimization, the maximum charging power $p_{\max }$ delivered by the charging infrastructure imposes a bound constraint to all elements of the optimization variable $P$.

$$
0 \leq p_{i, j} \leq p_{\max } \quad\left\{\begin{array}{l}
\mathrm{i}=1,2 \ldots, \mathrm{n} \\
\mathrm{j}=1,2, \ldots, \mathrm{m}
\end{array}\right\}
$$

The charging infrastructure subscribed power $p_{\text {subscribed }}$ imposes an inequality constraint to the submatrix $P_{\{j\}}$.

$$
A \times P_{\{j\}} \leq p_{\text {subscribed }}
$$

A denotes an all-ones $(1 \times \mathrm{n})$ matrix, $P_{\{j\}}$ is an $(\mathrm{n} \times 1)$ refers to the charging power for all the buses in a time slot $\mathrm{j}$.

The operating bus constraints (the bus arrival and departure time) impose an equality constraint to the submatrix $P_{\{i\}}$.

$$
B \times P_{\{i\}}^{T}=0
$$

B denotes an all-ones $(1 \times \mathrm{m})$ matrix that will take 0 between the bus arrival and departure time, $P_{\{i\}}^{T}$ (the transpose of the matrix $\left.P_{\{\boldsymbol{i}\}}\right)$ is an $(\mathrm{m} \times 1)$ matrix that represents the charging power of EB number I during the total time slots. 
The operating bus constraint (number of $\mathrm{km}$ to be covered during the following day) will define the final battery SoC to reach. It also imposes the amount of energy required to reach the targeted SoC as an equality constraint to the submatrix $P_{\{i\}}$.

$$
C \times P_{\{i\}}^{T}=\frac{\left(S o C_{\text {final }(i)}-S o C_{\text {initial }(i)}\right) \times Q_{\text {bat }(i)} \times V_{\text {bat }(i)}}{100 \times \Delta T \times \eta_{\operatorname{ch}(i)} \times \eta_{\text {bat }(i)}}
$$

C denotes an all-ones $(1 \times \mathrm{m})$ matrix, $P_{\{i\}}{ }^{T}$ is an $(\mathrm{m} \times 1)$ matrix that represents the total charging power of $\mathrm{EB}$ number $i(\mathrm{~W}), S o C_{\text {final }(i)}$ is the final state of charge of EB number $\mathrm{i}(\%), S o C_{\text {initial }(i)}$ is the initial state of charge of EB number i (\%), $\Delta T$ the time-slot of $30 \mathrm{~min}(\mathrm{~h}), Q_{\text {bat }(i)}$ denotes the total battery capacity of EB number $i(\mathrm{Ah}), \eta_{c h(i)}$ is the average charger efficiency of EB number $i(\%), \eta_{b a t(i)}$ is the average battery efficiency of EB number $i(\%), V_{b a t(i)}$ the average battery voltage of EB number $i(\mathrm{~V})$. The average values are calculated during a pre-optimization process which provides gross values of the optimization constraints.

\section{$\underline{\text { Nonlinear equality and inequality constraints }}$}

The charging process of a lithium-ion battery contains a constant current phase and a final constant voltage $(\mathrm{CV})$ phase dedicated to the end of the charging process. Considering the slow charging rate used in the case studied, we assumed that the $\mathrm{CV}$ phase lasts one hour (2 slots of $30 \mathrm{~min}$ ). As the $\mathrm{CV}$ phase is used to limit the current, we set the power in such a way that the $\mathrm{CV}$ phase charging allows the $\mathrm{SoC}$ to increase from 95 to $100 \%$ during two slots of $30 \mathrm{~min}$. As a first step, the end of charging process is not included on the optimization results facilitating a better understanding of the results.

\section{CASE STUDY}

A case study was performed to illustrate the optimization of an EBs fleet. The EBs fleet will operate during the day an existing conventional bus line. The optimization takes place during the night when the EB fleet returns to the depot. The EBs fleet is charged only at the depot during a period of 13.5 hours (from $t_{0}$ to $t_{0}+14 \mathrm{~h}$ ) divided into 27 time-slots of 30 min each, which results in a reasonable search space. The battery price was set at $500 € . \mathrm{kWh}^{-1}$ [22]. Table I presents the simulation parameters:

TABLE I. SIMULATION PARAMETERS OF THE OPTIMIZATION PROBLEM

\begin{tabular}{|l|r|}
\hline Parameters & Value / Specification \\
\hline Number of buses & 2 \\
\hline Number of simulated days & $1 \mathrm{day}$ \\
\hline Charging time period & $13.5 \mathrm{~h}$ \\
\hline Charging slot $\Delta t$ & $30 \mathrm{~min}$ \\
\hline Charging power for 1 charger & $P_{\max }$ \\
\hline Number of charging time slots & 27 \\
\hline Initial state of charge & $10 \%$ \\
\hline Initial battery temperature & $25{ }^{\circ} \mathrm{C}$ \\
\hline Initial battery capacity loss & $0 \%$ \\
\hline Fixed outside temperature & $25^{\circ} \mathrm{C}$ \\
\hline
\end{tabular}

The battery pack electro-thermal and aging characteristics are presented in Table II.

TABLE II. BATTERY ELECTRO-THERMAL AGING CHARACTERISTICS

\begin{tabular}{|c|c|}
\hline \multicolumn{2}{|l|}{ Parameters } \\
\hline Battery type & LIFePO4 or LFP \\
\hline Nominal energy/capacity & $311 \mathrm{kWh} / 540 \mathrm{Ah}$ \\
\hline Pack surface for thermal exchange & $18.79 \mathrm{~m}^{2}$ \\
\hline Weight & $2500 \mathrm{~kg}$ \\
\hline Specific heat capacity & $900 \mathrm{~J}^{\mathrm{kg}} \mathrm{kg}^{-1} \cdot \mathrm{K}^{-1}$ \\
\hline Heat transfer coefficient & $5 \mathrm{~W} \cdot \mathrm{m}^{-2} \cdot \mathrm{K}^{-1}$ \\
\hline A : pre-exponential factor & $4.35 \times 10^{7}$ p.u.day ${ }^{-1}$ \\
\hline$E_{a}$ : activation energy & $0.719 \mathrm{eV}$ \\
\hline $\mathrm{K}$ : Boltzmann constant & $8.617 \times 10^{-5} \mathrm{eV} . \mathrm{K}^{-1}$ \\
\hline B : quantity of charge factor & 1.104 \\
\hline $\mathrm{Q}_{\mathrm{EoL}}$ : Capacity loss at end of life & 0.2 p.u \\
\hline Bat $_{\text {price }}$ : Battery price & $500 € . \mathrm{kWh}^{-1}$ \\
\hline
\end{tabular}

The optimization constraints are shown in Table III.

TABLE III. EBS FLEET OPERATING CONSTRAINTS FOT TWO DAYS

\begin{tabular}{|l|l|l|l|}
\hline \multirow{2}{*}{\multicolumn{2}{|l|}{ Parameters }} & \multicolumn{2}{l|}{ Number of bus fleet } \\
\cline { 3 - 4 } \multicolumn{2}{l|}{ Initial SoC } & EB 1 & EB 2 \\
\hline Targeted SoC & Day j & $10 \%$ & $13 \%$ \\
\hline Arrival time & Day j & $100 \%$ & $40 \%$ \\
\hline Departure time & Day j+1 & $07: 30$ & $05: 00$ \\
\hline Length of the trip & Day j+1 & $179 \mathrm{~km}$ & $66 \mathrm{~km}$ \\
\hline Maximum charging power & & $60 \mathrm{~kW}$ & $60 \mathrm{~kW}$ \\
\hline Subscribed power & & \multicolumn{2}{|c|}{$100 \mathrm{~kW}$} \\
\hline
\end{tabular}

\section{RESULTS AND DISCUSSIONS}

This section presents some results of the proposed method applied to the considered case study. In a first step, we focus on mono-objective optimization of a fleet of 2 EBs with variant operating constraints by minimizing the electricity $\operatorname{cost}\left(\alpha_{1}=1, \alpha_{2}=0\right)$ or the battery aging $\operatorname{cost}\left(\alpha_{1}=0, \alpha_{2}=1\right)$.

As a final step, a multi-objective optimization of the charging and battery aging cost will be performed with a Pareto front representation using variable $\alpha_{1}, \alpha_{2}$ values. The Pareto front optimization results will be compared to the NSGA-II results. To illustrate the potential economic gain, we will compare our optimal strategy to a "Greedy" baseline method, which represents one typical behavior that charges the EB with the maximum power as soon as possible, ignoring electricity and battery aging cost, until it is fully charged. 


\section{A. Mono-objective optimization of the electricity cost for an} EBs fleet charging $\left(\alpha_{1}=1, \alpha_{2}=0\right)$

The results of mono-objective optimization for minimizing electricity cost in Fig. 4 regardless of the battery aging show that the optimal charging power (EB1, EB2) agrees perfectly with the variation of the electricity cost while respecting operating constraints for both EBs. In addition, the algorithm ensures that the total charging power will not exceed the subscribed one.
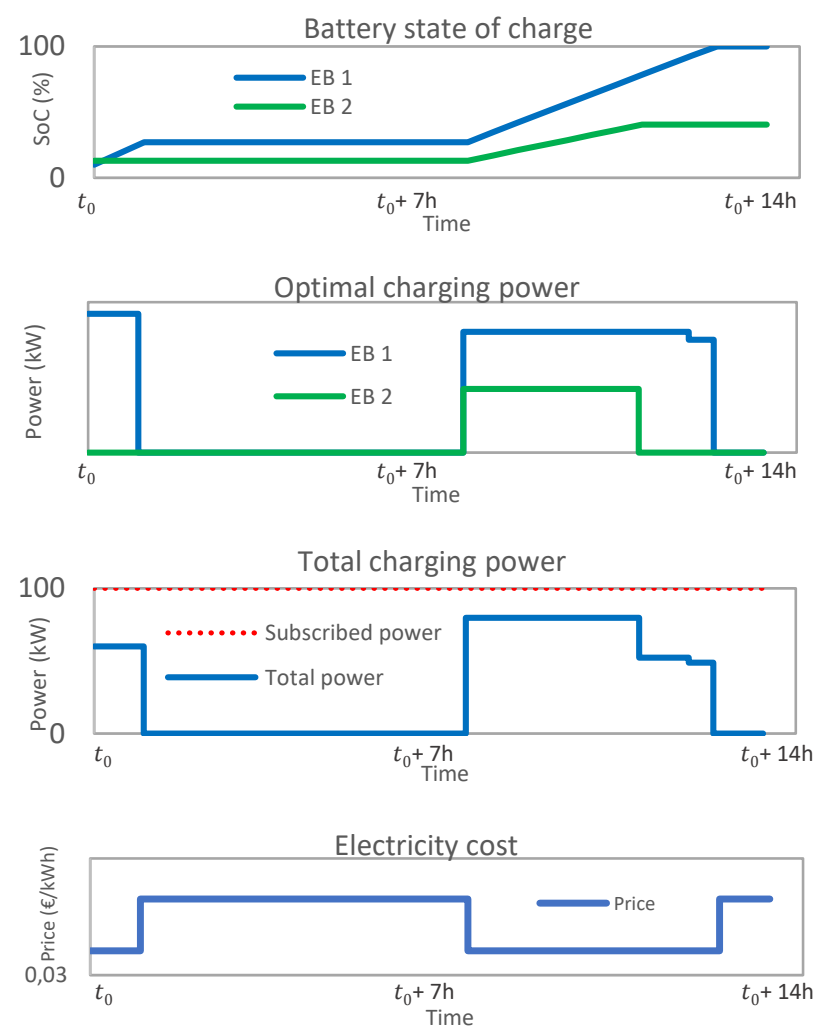

Fig. 4. Optimal charging power to minimize electricity cost of two EBs

\section{B. Mono-objective optimization of the aging cost for one EBs fleet charging $\left(\alpha_{1}=0, \alpha_{2}=1\right)$}

The results of mono-objective optimization for minimizing battery aging cost in Fig. 5 show that the optimal charging power (EB1, EB2) tends to increase gradually. This seems logical according to the aging fitness function defined in (4). The optimal charging power profile decides to delay the charge to minimize calendar aging due to higher values of $\mathrm{SoC}$ and gradually increases the power to avoid power peaks that will cause battery overheating. The EB2 starts charging late and stops charging earlier due to the operating constraints. It can be noticed that the EB2 does not reach its maximum power to not exceed the subscribed power.
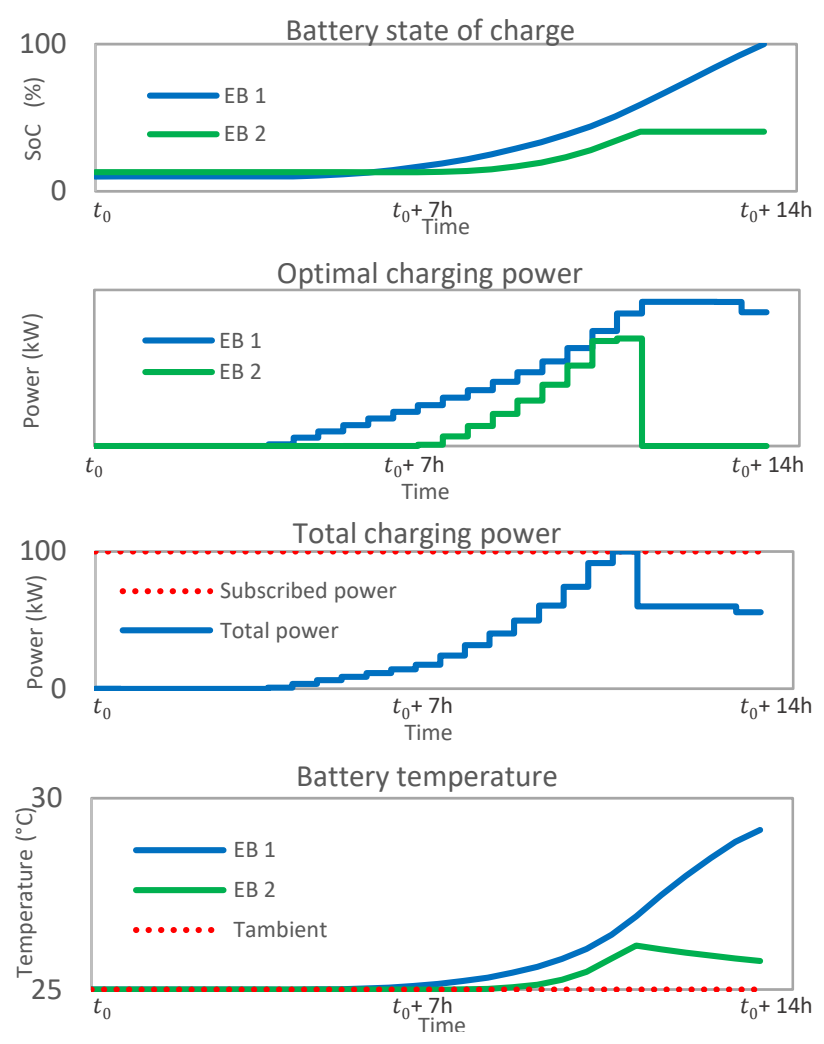

Fig. 5. Optimal charging power to minimize battery aging cost of two EBs

\section{Multi-objective optimization of both electricity and} aging cost for one EB charging

The results of NLP multi-objective optimization are compared to NSGA-II algrotihm results and to a Greedy baseline solution in Fig. 6. The Pareto front expresses the trade-off between these two competing objectives and proposes different optimal solutions. NSGA-II Pareto front comes close to NLP and explore a much larger area but is not able to converge well particularly for the battery aging cost objective. This is due to the limit of the optimization variable that is discrete in the NSGA-II problem optimization (more details in [12]) and continuous in NLP optimization.

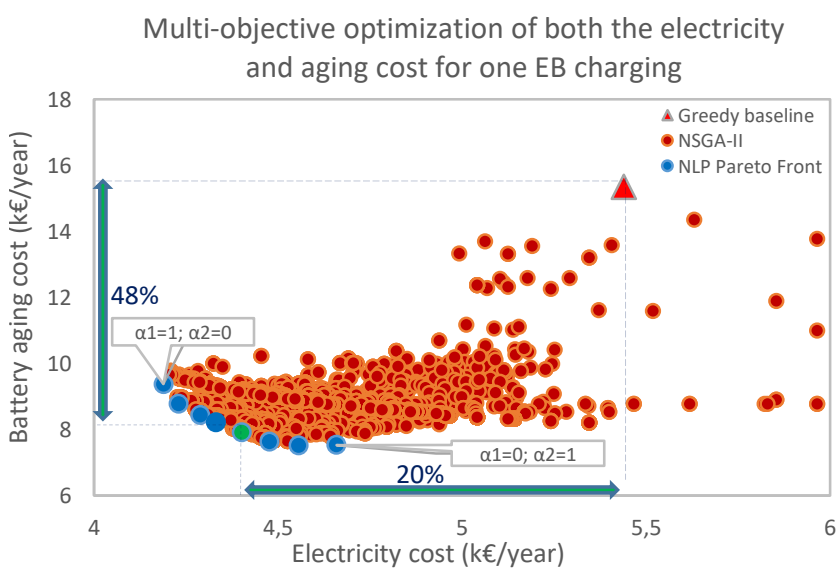

Fig. 6. Multi-objective optimization of both the electricity and aging cost for one EB charging 
The results of the simulation show that a potential solution from the Pareto front that satisfies both objectives has been able to achieve a $20 \%$ reduction in the annual electricity cost as well as a $48 \%$ reduction in the battery aging cost compared to the greedy baseline solution.

\section{CONCLUSION AND FUTURE WORKS}

This paper introduces a smart charging method for an EB fleet. A case study has been presented and analyzed. The implemented optimization algorithm namely NLP achieves good performance after only 2 minutes of computing time for one EB that makes possible to deal with large fleet of several hundred buses. The comparison between the two methods showed that NSGA-II explored a larger area of potential solutions but does not perform very well in some regions while consuming much more CPU processing time.

In future work, the purpose is to deal with a larger problem with different sizes of bus fleets to see how the algorithm performs. The end of the charging process will be also included as it constrains the available optimization period. Particular attention will be paid to include the battery cycling aging mainly during the charge at cold temperatures. The battery thermal model sensitivity has to be carefully studied.

\section{REFERENCES}

[1] M. Dietmannsberger, "Modelling the Electrification of Bus Depots using Real Data: Consequences for the Distribution Grid and Operational Requirements," 1st E-Mobility Power System Integration Symposium, p. 8, 2017.

[2] J. Hu, H. Morais, T. Sousa, and M. Lind, 'Electric vehicle fleet management in smart grids: A review of services, optimization and control aspects', Renewable and Sustainable Energy Reviews, vol. 56, no. Supplement C, pp. 1207-1226, Apr. 2016.

[3] M. Rinaldi, F. Parisi, G. Laskaris, A. D'Ariano, and F. Viti, "Optimal dispatching of electric and hybrid buses subject to scheduling and charging constraints," in 2018 21st International Conference on Intelligent Transportation Systems (ITSC), 2018, pp. 41-46.

[4] Y. Gao, S. Guo, J. Ren, Z. Zhao, A. Ehsan, and Y. Zheng, "An Electric Bus Power Consumption Model and Optimization of Charging Scheduling Concerning Multi-External Factors," Energies, vol. 11, no. 8, p. 2060, Aug. 2018.

[5] A. Jahic, M. Eskander, and D. Schulz, "Charging Schedule for Load Peak Minimization on Large-Scale Electric Bus Depots," Appl. Sci., vol. 9, no. 9, p. 1748, Apr. 2019.

[6] R. Guyot, B. Lasserre, J.-L. Vicente, L. Torcheux and L. Martin, "Use of data for electric transport network optimization. Optimal charging at bus depots," Electric Vehicle Symposium 32, Lyon, 2019.

[7] M. Jiang et al., "Operation and Scheduling of Pure Electric Buses under Regular Charging Mode," in 201821 st International Conference on Intelligent Transportation Systems (ITSC), 2018, pp. 1894-1899.

[8] R.-C. Leou and J.-J. Hung, "Optimal Charging Schedule Planning and Economic Analysis for Electric Bus Charging Stations," Energies, vol. 10, no. 4, p. 483, Apr. 2017.

[9] Huimiao Chen et al., "Coordinated charging strategies for electric bus fast charging stations," in 2016 IEEE PES Asia-Pacific Power and Energy Engineering Conference (APPEEC), 2016, pp. 1174-1179.

[10] G. Wang, X. Xie, F. Zhang, Y. Liu, and D. Zhang, "bCharge: DataDriven Real-Time Charging Scheduling for Large-Scale Electric Bus Fleets," in 2018 IEEE Real-Time Systems Symposium (RTSS), 2018, pp. $45-55$.

[11] T. Zhu et al., "An Optimized Energy Management Strategy for Preheating Vehicle-Mounted Li-ion Batteries at Subzero Temperatures," Energies, vol. 10, no. 2, p. 243, Feb. 2017.
[12] A. Houbbadi, R. Trigui, S. Pelissier, E. Redondo-Iglesias, and T. Bouton, "Multi-Objective Optimisation of the Management of Electric Bus Fleet Charging," in 2017 IEEE Vehicle Power and Propulsion Conference (VPPC), Belfort, 2017, pp. 1-6.

[13] Project KPI presentation sample by YouExec https://fr.slideshare.net/imokymas/project-kpi-presentation-sampleby-youexec.

[14] R. Trigui, B. Jeanneret, and F. Badin, "Systemic modelling of hybrid vehicles in order to predict dynamic performance and energy consumption building the VEHLIB library of models," RTS, vol. 21, no. 83, pp. 129-150, 2004.

[15] J. Jaguemont, L. Boulon, and Y. Dube, "Characterization and Modeling of a Hybrid-Electric-Vehicle Lithium-Ion Battery Pack at Low Temperatures," IEEE Trans. Veh. Technol., vol. 65, no. 1, pp. 114, Jan. 2016.

[16] A. Houbbadi, R. Trigui, S. Pelissier, E. Redondo-Iglesias, and T. Bouton, "Optimal scheduling to manage an electric bus fleet overnight charging," Energies, vol. 12, no. 14, p. 2727, Jan. 2019.

[17] M. Petit, E. Prada, and V. Sauvant-Moynot, "Development of an empirical aging model for $\mathrm{Li}$-ion batteries and application to assess the impact of Vehicle-to-Grid strategies on battery lifetime," Appl. Energy, vol. 172, pp. 398-407, Jun. 2016.

[18] E. Redondo-Iglesias, P. Venet, and S. Pelissier, "Eyring acceleration model for predicting calendar ageing of lithium-ion batteries," Journal of Energy Storage, 2017, 13, 176 - 183.

[19] H. Tamaki, H. Kita, and S. Kobayashi, "Multi-objective optimization by genetic algorithms: a review," in Proceedings of IEEE International Conference on Evolutionary Computation, 1996, pp. 517-522.

[20] X. Wang, F. Dugardin, and F. Yalaoui, "An exact method to solve a Bi-objective Resource Constraint Project Scheduling Problem," IFACPap., vol. 49, no. 12, pp. 1038-1043, Jan. 2016.

[21] N. Jozefowiez, "Multi-objective combinatorial optimization: From methods to problems, from the Earth to (almost) the Moon," thesis, Institut National Polytechnique de Toulouse (INP Toulouse), 2013.

[22] Pillot, C. Lithium ion battery raw material supply \& demand 20162025. Avicenne Energy http://cii-resource.com/cet/AABE-0317/Presentations/BRMT/Pillot_Christophe.pdf (2017). 